\title{
Comparative Analysis of Surrogacy in the Northeast region of the U.S
}

\section{Ana Arias-Oliveras}

School of Nursing, University of Pennsylvania, New Jersey, USA

*Corresponding author: Ana Arias-Oliveras, University of Pennsylvania, School of Nursing, Philadelphia PA 3021 Main Street Voorhees, New Jersey, 08043, USA, Tel: +1 215-898-8281; E-mail: ariasai@upenn.edu

Received date: September 07, 2017; Accepted date: October 04, 2017; Published date: October 12, 2017

Copyright: $\odot 2017$ Arias-Oliveras A. This is an open-access article distributed under the terms of the Creative Commons Attribution License, which permits unrestricted use, distribution, and reproduction in any medium, provided the original author and source are credited.

\begin{abstract}
Advancement in the science of Artificial Reproductive Therapies (ART) has led to an increase of children born pursuant surrogacy arrangements. It is considered to be a form of family building for couples or individuals who are unable to carry a pregnancy. Within the United States, there are two surrogacy models, Gestational and Traditional. Gestational surrogacy is when the intended parents obtain a surrogate host who will carry the fetus until delivery. As part of the agreement, the surrogate relinquishes parental rights prior to the birth of the child. Traditional surrogacy is when the intended parents obtain a surrogate host who will agree to provide an ovum and carry the fetus until delivery. The embryo is a product of the surrogate and the husband of the commissioning couple, thus creating a genetic link between the surrogate and child. Parental rights are relinquished by the surrogate at the time of delivery, but due to the variability in adoption laws, may not be official for up to several years. Surrogacy as a viable option for infertile couples should be taken into consideration when one of the following circumstances are present: it is biologically impossible for one or both intended parents and/or is medically contraindicated to carry a baby regardless if this condition is congenital or acquired.
\end{abstract}

Keywords: Artificial reproductive therapies; Surrogacy; Congenital; Gynaecologists; Obstetricians

\section{Introduction}

The American College of Obstetricians and Gynaecologists (ACOG) has provided recommendations to ensure all aspects of Gestational Surrogacy are considered and upheld to public policy standards, including state legislation. Both the Obstetrician and legal representatives should be well versed in all aspects of gestational surrogacy (medical, ethical and psychosocial complexities) in addition to the legal statutes in the states they practice. The intended couple and surrogate should have separate, legal counsel and separate independent mental health screenings [1]. It is imperative that all parties, including the Obstetrician comply with state legislation to avoid any potential legal ramifications secondary to noncompliance to surrogacy laws. Aspects of surrogacy that will need to be considered: domicile of intended parents, domicile of surrogate, state where surrogate undergoes in vitro fertilization (IVF), state where surrogate will deliver child and state where the gestational surrogate agreement will be submitted to the court.

The American Society of Reproductive Medicine (ASRM) has provided recommendations to provide guidance for considerations of the use of a surrogate in addition to the screening practices of both the intended parents and surrogate. These guidelines have been developed to minimize the possibility of complications that may arise and to address any medical and/or psychosocial complexities that may affect all parties, including the children. The impact of relinquishing a child following birth can have emotional consequences for the surrogate, thereby obtaining a thorough understanding of surrogate mothers' psychological functioning is imperative [2]. One of the landmark cases, Annas suggested that state law should require all parties in a surrogacy agreement must meet the following criteria (where applicable) undergo a psychological evaluation; the genetic mother is unable to carry a child to term, the surrogate must relinquish parental rights in addition to she must have previously given birth to at least one child [3].

\section{Types of Surrogacy}

Gestational surrogacy is where the intended parents obtain a surrogate as a host for their embryo. The surrogate will undergo IVF to be implanted with an embryo that is genetically linked to the intended parents. An agreement will be completed prior to the initiation of the IVF process. There are many aspects of the contract that will need to be discussed in detail, but the most important is the understanding that the surrogate does not have any parental rights to this child. At no time during the pregnancy or immediately following the birth of child, does the surrogate have the right to decide on behalf of the foetus. It is highly recommended that the parties involved complete a genetic, medical and psychosocial evaluation to determine if this will result in a successful transition from surrogate to family.

Traditional surrogacy is when the surrogate provides the ovum creating a biological link to the foetus. The surrogate undergoes artificial insemination with the sperm provided by the male partner of the intended couple. The surrogate is considered the mother of the child and agrees to relinquish her parental rights to the child once born. Adoption laws are variable state to state, so the length of time for the adoption process to be finalized is undetermined at the time the agreement is signed. Due to these circumstances, most states prohibit this type of surrogacy. Parental disputes are more likely in this model versus gestational surrogacy.

\section{Legislation and Regulation of Surrogacy Laws}

Under the current framework, there exist three policy options in which a state may adopt in regulating surrogacy parenting contracts [4]. The state legislation can be categorized as follows: Nonenforcement, Prohibition and Regulated Enforcement. Non- 
enforcement suggests that the individual state does not have surrogacy laws in place. Although current legislation does not regulate surrogacy, the following states have courts in favour of surrogacy arrangements: Maryland, Massachusetts, Pennsylvania, Rhode Island and Vermont. Individual states may have certain applicable criteria for honouring a gestational surrogacy contract such as: one of the intended parents must provide a gamete for the embryo that is to be implanted, compensatory contracts are prohibited and only gestational surrogacy is permitted.

Prohibition of surrogacy (both types) is active in both New York and Washington D.C. New York had legislation in favour of surrogacy until the case of Matter of Adoption of Baby Girl L.J. 1998 catapulted a change in surrogacy laws.

Regulated enforcement is considered when states provide surrogacy laws to guide intended parents, surrogates, Obstetricians and legal representatives. For example, Connecticut permits both traditional and gestational surrogacy where as New Hampshire allows gestational contracts for heterosexual couples with the provision, that one provides the gamete for the embryo.

The recommendations and guidelines set forth by the ACOG, the ASRM and the Parentage Act do not supersede state laws. It is imperative that all parties involved in a surrogate arrangement follow the standards stipulated by the state where the contract is legalized and enforced. Significant variability exists state to state regarding surrogacy laws [5]. Some states, such as New York, consider both traditional and gestational surrogacy illegal; therefore all participants may be subject to criminal penalties.

\section{Ethical Considerations}

There is a growing concern that Surrogacy has become commercialized to the point of perceiving it as "renting a womb" or "motherhood for sale" [6]. Should compensation be considered for both types of surrogacy? If so, to what extent? State to state variability on this aspect has generated discussions on regulation of compensation or prohibiting it altogether. Should there be a distinction between providing a surrogate receives compensation for travel, medications, loss of wages during pregnancy, at the time of delivery and for the post-partum period, versus allocating a predetermined sum of money at the time the child is delivered.

Concerns for comparison between domestic and international surrogacy include: cost of surrogacy to term gestation, legitimacy of agreements, amount of compensation expected (depends on the country and state where contracts are legitimate), rate of successful implantation and gestating until term [7]. There is an increase in the use of international surrogates due to the cost in comparison to the use of domestic surrogacy.

\section{Special Considerations}

It is highly recommended that all participants discuss potential circumstances that may arise during the pregnancy. These discussions should occur with all participants and in the presence of the individual legal representatives. I will present some cases and provide an overview based on the surrogacy model:

\section{Case 1}

It is noted on a Level 2 ultrasound that the foetus has multiple congenital anomalies. The intended parents request that the surrogate undergoes an abortion.

\section{Gestational surrogacy model}

Although the surrogate relinquishes her parental rights prior to undergoing IVF, she may decline the request of the intended couple. Due to the variability of surrogacy laws state to state, the surrogacy agreement is not enforceable outside of state limits. For example, if the intended parents live in New York, the surrogate was implanted with the embryo in Connecticut, but lives in Pennsylvania, the surrogate may move to Washington D.C where surrogacy is considered illegal, therefore the agreement is not enforceable. If the intended couple pursues legal action against the surrogate for violating the contract, the courts have used several cases depicting "the best interest" of the child. The American Academy of Paediatrics has recommended the best interest standard of the child as an important standard. Determining the best-interest of the child requires careful analysis of both the child and families involved [8].

\section{Traditional surrogacy model}

The surrogate is the biological mother. Most states will honour her decision to either accept or decline the request of the intended couple. A stipulation that may be viewed as a complication, is if the surrogate is married, then the surrogate and her husband have parental rights that can be enforced throughout the pregnancy up until the adoption is formalized via court.

\section{Case 2}

A surrogate presents to Labor and Delivery at 23 weeks gestation. She is in active labor and has bulging membrane on exam.

\section{Gestational surrogacy model}

The surrogate does not have the right to decide on behalf of the foetus in this instance, unless there is a specific stipulation in the agreement. Another consideration is if the surrogate happens to deliver in a state where the agreement is not enforceable, then she may decide on behalf of the foetus. The intended parents have the parental rights once the child is born and can consent to treatment or decline further interventions.

\section{Traditional surrogacy model}

The surrogate is the biological mother and has the right to make the decision on behalf of the foetus. Whether it is to forgo medical interventions or not.

\section{Case 3}

A surrogate undergoes an anatomy scan around 20 week's gestation. It is revealed that the foetus has a myelomeningocele at the level of L4 and has findings consistent with hindbrain herniation. The intended couple and surrogate seek a foetal consult at their local Foetal Center. All parties participate in the consult and it is determined that the surrogate and foetus meet criteria for foetal surgery.

Under both models, can the intended couple enforce the contract so that the surrogate undergoes foetal surgery? Is it ethical to dismiss the 
Citation: Arias-Oliveras A (2017) Comparative Analysis of Surrogacy in the Northeast region of the U.S. J Nurs Care 6: 428. doi: $10.4172 / 2167-1168.1000428$

Page 3 of 3

surrogate's right to autonomy? If we are to consider the other ethical rights such as Beneficence, Justice and Non-maleficence, do we consider the surrogate, the foetus, the intended parents or all?

\section{Autonomy}

In both models, the surrogate has a right to Autonomy; therefore she may decline to undergo foetal surgery. All surrogacy arrangements take into consideration foetal health and well-being. The surrogate is under no obligation to subject herself to medical treatment she is not in agreement with.

\section{Beneficence}

How do you balance the autonomy of the surrogate over the best interest of the foetus in this case?

\section{Non-maleficence}

Undergoing foetal surgery has its risks for the both the surrogate and foetus. How do we determine the risk benefit ratio and for whom?

\section{Justice}

Based on the MOMs trial, we know that there is some long-term benefit to undergo foetal surgery for the diagnosis of myelomeningocele. The prognosis is beneficial not only to the foetus, but improved outcomes

\section{Discussion and Conclusion}

It is imperative that both the intended parents and surrogate follow the recommendations and guidelines provided by ACOG and ASRM to decrease the possibility of complications that may arise during or after the pregnancy. Legal counsel obtained for both parties should be experienced and knowledgeable in the current state's surrogacy laws to ensure compliance and avoid any legal sanctions. The intended parents should evaluate all their options prior to committing to a surrogacy contract. Consider the state laws that govern surrogacy not only in their place of domicile, but also where the surrogate lives, where she will deliver their child, where and how the adoption process will take place. Other considerations that will decrease the likelihood of conflict is to ensure a detailed discussion of potential maternal and foetal circumstance that may arise during the pregnancy. Full disclosure and awareness by all parties will enhance the transition from surrogacy to family.

\section{References}

1. American College of Obstetricians and Gynecologists (2016) Family building through gestational surrogacy committee on ethics, Number 660.

2. Pizits TD, McCullough J, Rabin A (2013) Do women who choose to become surrogate mothers have different psychological profiles compared to a normal female sample. Women Birth 26: e15-20.

3. Annas GJ (1991) Determining the fate of gestational mothers. Womens Health Issues 1: 158-160.

4. Hill JL (1990) The case for enforcement of the surrogate contact. Politics and the Life Sciences 8: 147-160.

5. James S, Chilvers R, Havemann D, Phelps JY (2010) Avoiding legal pitfalls in surrogacy arrangements. Reprod Biomed Online 21: 862-867.

6. Bulfin MJ (1991) Surrogate motherhood. Women's Health Issue 1: 140-142.

7. Banerhee S, Basu S (2009) Rent a womb: Surrogate selection, investment incentives and contracting. J Econ Behav Organ 69: 260-273.

8. American Academy of Pediatrics (1991) Ethical issues in surrogate motherhood. Committee on Bioethics. 